\title{
Proton momentum distributions and elastic electron scattering form factors for some Ge isotopes
}

\author{
Al- Rahmani A. A., Kaddoori F. F. \\ Department of Physics, College of Science for Woman, University of Baghdad, \\ Baghdad, Iraq \\ E-mail: a.alrehmani@yahoo.com
}

\begin{abstract}
The proton momentum distributions (PMD) and the elastic electron scattering form factors $\mathrm{F}(\mathrm{q})$ of the ground state for some even mass nuclei in the 2p-1f shell for ${ }^{70} \mathrm{Ge},{ }^{72} \mathrm{Ge},{ }^{74} \mathrm{Ge}$ and ${ }^{76} \mathrm{Ge}$ are calculated by using the Coherent Density Fluctuation Model (CDFM) and expressed in terms of the fluctuation function (weight function) $|\mathrm{F}(\mathrm{x})|^{2}$. The fluctuation function has been related to the charge density distribution (CDD) of the nuclei and determined from the theory and experiment. The property of the long-tail behavior at high momentum region of the proton momentum distribution has been obtained by both the theoretical and experimental fluctuation functions. The calculated form factors $F(q)$ of all nuclei under study are in good agreement with those of experimental data throughout all values of momentum transfer $\mathrm{q}$.
\end{abstract}

Key words

Density distributions, elastic electron scattering form factors, momentum distributions, $2 p-1 f$ shell nuclei, Coherent Density Fluctuation Model (CDFM).

\section{Article info.}

Received: Oct. 2016

Accepted: Nov. 2016

Published: Mar. 2017

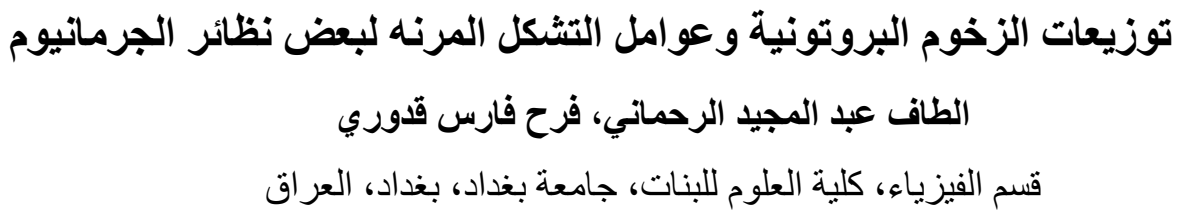

نم حساب كل من توزيعات زخم البروتونات وعوامل التشكل للاسنطارة الالكترونيه المرنة للحالة الارضية

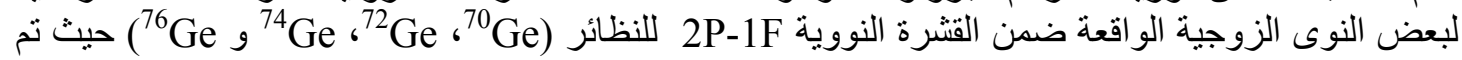

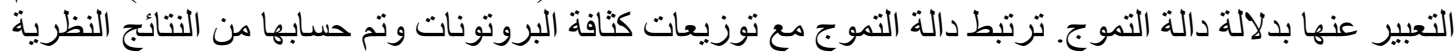

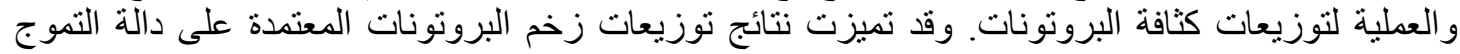

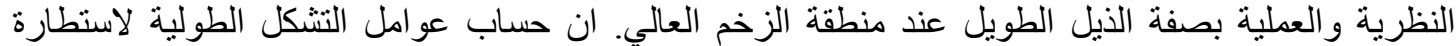
الالكترون المرنة لجميع النوى النظرية تتفق مع النتائج العملية للنوى.

\section{Introduction}

The study of momentum distribution is important tool for studying the ground state properties of nuclei, especially the momentum distribution of protons [1]. This is measured in the framework of the coherent density fluctuation model (CDFM), which is exemplified by the work of Antonov et al. [2, 3]. There is no method for directly measuring the proton momentum distribution PMD in nuclei. The quantities that are measured by particle-nucleus and nucleus-nucleus collisions are the cross sections of different reactions, and these contain information on the PMD of target nucleus. The experimental evidence obtained from inclusive and exclusive electron scattering on nuclei establish the existence of long- tail behavior of the PMD at high momentum region $\left(\mathrm{k} \geq 2 \mathrm{fm}^{-1}\right)$ [4-6]. The mean field theories cannot 
describe correctly the form factor $\mathrm{F}(\mathrm{q})$ and the PMD simultaneously [7] and they exhibit a steep- slope behavior of the PMD at high momentum region. In fact, the PMD depends a little on the effective mean field considered due to its sensitivity to the short rang and tensor nucleon-nucleon correlations [7, 8] which are not included in the mean field theories. In the CDFM, the local charge density distribution (CDD) and the PMD are simply related and expressed in terms of an experimentally obtainable fluctuation function (weight function) $|f(x)|^{2}$. A lot of experimental and theoretical work on elastic and inelastic electron scattering at different energies has provided detailed information on the charge density distribution of the nuclear ground state and on the energy, strength, and quantum numbers of the excited states produced by single particle or collective excitation mechanisms [9-12]. The interest in charge densities result from that, they can provide more detailed information for the internal structure of nuclei, because they are directly related to the wave functions of protons that is important keys for many calculations in nuclear physics $[13,14]$. There are several theoretical methods used to study elastic electron- nucleus scattering, such as the plan-wave Born approximation (PWBA), the eikonal approximation and the phase-shift analysis method [15-19]. The PWBA method can give qualitative results and has been used widely for its simplicity. To include the Coulumb distortion effect, which is neglected in PWBA, the other two methods may be used. In the last few years, some theoretical studies of elastic electron scattering off exotic nuclei have been performed. Wang et al. [15, 16] studied such scattering along some isotopic and isotonic chains by combining the eikonal approximation with the relativistic mean field theory. Karataglidis and Amos [18] have studied the elastic electron scattering form factors, longitudinal and transverse, from exotic ( $\mathrm{He}$ and $\mathrm{Li}$ ) isotopes and from $\mathrm{B}$ nucleus using large space shell models. Al-Rahmani and Hussien [20] have studied the CDD and elastic electron scattering form factors of some $2 \mathrm{~s}-1 \mathrm{~d}$ shell nuclei using the PWBA and demonstrated that the inclusion of the higher $1 f-2 p$ shell in the calculation leads to produce a good result in comparison with those of the experimental data. Hamoudi et al. [21] have been calculated elastic electron scattering form factor (EESFF) and the nucleon momentum distribution (NMD) of the ground state for $p$-shell nuclei with $\mathrm{Z}=\mathrm{N}$ such as $\left({ }^{6} \mathrm{Li},{ }^{10} \mathrm{~B},{ }^{12} \mathrm{C}\right.$ and ${ }^{14} \mathrm{~N}$ nuclei). Besides, the weight functions have expressed in terms of nucleon density distribution of the nuclei and the coherent density fluctuation model has expressed in term weight function $|f(x)|^{2}$ and measured from the experiment and the theory. Their results appeared a good agreement with the experimental results. ALRahmani A.A. [22] have been calculated the G.S. elastic charge form factors and proton momentum distribution for the upper region of the $2 \mathrm{~S}-1 \mathrm{~d}$ shell nuclei like $\left({ }^{35} \mathrm{CI},{ }^{37} \mathrm{CI}\right.$ and ${ }^{39} \mathrm{~K}$ ). At the same year, also, ALRahmani A.A. [22] have measured the nucleon momentum distributions and elastic electron scattering form factor of the ground state for some odd $2 \mathrm{~s}-1 \mathrm{~d}$ shell nuclei like $\left({ }^{19} \mathrm{~F},{ }^{25} \mathrm{Mg},{ }^{27} \mathrm{Al}\right.$ and ${ }^{29} \mathrm{Si}$ ) by using the coherent density fluctuation model and expressed in terms of the fluctuation function (weight function) $|\mathrm{f}(\mathrm{x})|^{2}$. In addition, through her works she found that the inclusion of the quadrapole form factors $\mathrm{F}_{\mathrm{C} 2}(\mathrm{q})$ in all nuclei under study which was described by the undeformed 2S-1d shell nuclei, was 
essential for obtained a notable accordance between the experimental and theoretical form factors. It is important to point out that all above calculations obtained in the framework of CDFM proved a high momentum tail in the PMD. Elastic electron scattering from ${ }^{40} \mathrm{Ca}$ nucleus was also investigated in [23], where the calculated elastic differential cross sections $d s / d \Omega$ are in good agreement with those of experimental data.

The aim of the present work is to derive an analytical expression for the CDD applicable throughout all 2p-1f shell nuclei based on the use of the single particle harmonic oscillator wave functions and the occupation numbers of the states. The derived form of the CDD is employed in determining the theoretical weight function $|f(x)|^{2}$ which is then used in the CDFM to study the PMD and elastic scattering form factors $F(q)$ for some $2 p$-1f shell nuclei for ${ }^{70} \mathrm{Ge},{ }^{72} \mathrm{Ge}$, ${ }^{74} \mathrm{Ge}$ and ${ }^{76} \mathrm{Ge}$ isotopes. It is found that the theoretical weight function based on the derived CDD is capable to give information about the PMD and elastic charge form factors as do those of experimental data.

\section{Theory}

The charge density distribution of one -body operator can be written respectively, as [21]

$$
\rho_{c}(r)=\frac{1}{4 \pi} \sum_{n l} \zeta_{n l} 2(2 l+1)\left|R_{n l}(r)\right|^{2}
$$

where $\rho_{c}(r)$ is the charge density distribution of nuclei, $\zeta_{n l}$ is the proton occupation probability of the state $n l \quad\left(\zeta_{n l}=0\right.$ or 1 for closed shell nuclei and $0<\zeta_{n l}<1$ for open shell nuclei) and $R_{n l}(r)$ is the radial part of the single-particle harmonic oscillator wave function. To derive an explicit form for the CDD of $1 f-2 p$ shell nuclei, it is supposed that there is a core of filled $1 s$ and $1 p$ and $1 \mathrm{~d}$ shells and the proton occupation numbers in $2 s, 1 f$ and $2 p$ shells are equal to $(2-\alpha), \beta$ and $(Z-20-\beta+\alpha)$, respectively, for $\left({ }^{70} \mathrm{Ge},{ }^{72} \mathrm{Ge},{ }^{74} \mathrm{Ge}\right.$ and $\left.{ }^{76} \mathrm{Ge}\right)$, instead of $2,(Z-20)$ and 0 as in the simple shell model. Using this assumption in Eq. (1), we get:-

$\rho_{c}(r)=\frac{1}{4 \pi}\left\{2\left|R_{10}(r)\right|^{2}+6\left|R_{11}(r)\right|^{2}+10\left|R_{12}(r)\right|^{2}+(2-\alpha)\left|R_{20}\right|^{2}+\beta\left|R_{13}\right|^{2}+(Z-20-\beta+\alpha)\left|R_{21}\right|^{2}\right\}$

where $Z$ is the atomic number of nuclei, the parameter $\alpha$ characterizes the deviation of the proton occupation numbers from the prediction of the simple shell model $(\alpha=0)$, the parameter $\beta$ is assumed as a free parameter to be adjusted in order to obtain the agreement with the

$$
\begin{aligned}
\rho_{c}(r)=\frac{e^{-r^{2} / b^{2}}}{\pi^{3 / 2} b^{3}}\left\{\left(5-\frac{3}{2} \alpha\right)+\left[\frac{11}{3} \alpha+\frac{5}{3}(Z-20-\beta)\right]\left(\frac{r}{b}\right)^{2}+\left[4-2 \alpha-\frac{4}{3}(Z-20-\beta)\right]\left(\frac{r}{b}\right)^{4}\right. \\
\left.+\left[\frac{8}{105} \beta+\frac{4}{15}(Z-20-\beta)+\frac{4}{15} \alpha\right]\left(\frac{r}{b}\right)^{6}\right\}
\end{aligned}
$$


The mean square charge radius (MSR) of the considered $1 \mathrm{f}-2 \mathrm{p}$ shell nuclei can be written as: $[2,3]$

$$
\left\langle r^{2}\right\rangle=\frac{4 \pi}{Z} \int_{0}^{\infty} \rho_{c}(r) r^{4} d r
$$

The normalization condition of the $\rho_{c}(r)$ is given by $[2,3]$

$$
Z=4 \pi \int_{0}^{\infty} \rho_{c}(r) r^{2} d r
$$

And the corresponding MSR is

$$
\left\langle r^{2}\right\rangle=b^{2}\left\{\frac{9}{2}-\frac{30}{Z}+\frac{\alpha}{Z}\right\}
$$

The central $\rho_{c}(r=0)$ is obtained from Eq. (3) as

$$
\rho_{c}(0)=\frac{1}{\pi^{3 / 2} b^{3}}\left\{5-\frac{3}{2} \alpha\right\}
$$

The parameter $\alpha$ can be determined from the central CDD of Eq. (6) as

$\alpha=\frac{2}{3}\left\{5-\pi^{3 / 2} b^{3} \rho_{c}(0)\right\}$

In Eq. (8), the values of the central density, $\rho_{c}(0)$, are taken from the

$$
n(k)=\frac{b^{3} e^{-x^{2} / b^{2}}}{\pi^{3 / 2}}\left[\begin{array}{l}
5-\frac{3}{2} \alpha+\left\{\frac{11}{3} \alpha+\frac{5}{3}(Z-20-\beta)\right\}\left(\frac{x}{b}\right)^{2} \\
+\left\{4-2 \alpha_{1}-\frac{4}{3}(Z-20-\beta)\right\}\left(\frac{x}{b}\right)^{4} \\
\left.+\frac{8}{105} \beta+\frac{4}{15}(Z-20-\beta)+\frac{4}{15} \alpha\right\}\left(\frac{x}{b}\right)^{6}
\end{array}\right]
$$

while in the second method, the $n(k)$ can be determined by the CDFM, where the mixed density is given by $[2,3]$

$$
\rho\left(\vec{r}, \vec{r}^{\prime}\right)=\int_{0}^{\infty}|f(x)|^{2} \rho_{x}\left(\vec{r}, \vec{r}^{\prime}\right) d x
$$

since

$\rho_{x}\left(\vec{r}, \vec{r}^{\prime}\right)=3 \rho_{0}(x) \frac{j_{1}\left(k_{F}(x)\left|\vec{r}-\vec{r}^{\prime}\right|\right)}{k_{F}(x)\left|\vec{r}-\vec{r}^{\prime}\right|} \times \theta\left(\vec{x}-\frac{\left|\vec{r}+\vec{r}^{\prime}\right|}{2}\right)$

is the density matrix for $Z$ protons uniformly distributed in the sphere with radius $x$ and density

$$
k_{F}(x)=\left(\frac{3 \pi^{2}}{2} \rho_{0}(x)\right)^{1 / 3} \equiv \frac{V}{x} ; \quad V=\left(\frac{9 \pi Z}{8}\right)^{1 / 3}
$$
$\rho_{0}(x)=3 Z / 4 \pi x^{3}$. The Fermi momentum is defined as $[2,3]$

and the step function $\theta$, in Eq. (12), is defined by 


$$
\theta(y)= \begin{cases}1, & y \geq 0 \\ 0, & y<0\end{cases}
$$

According to the density matrix definition of Eq. (11), one-particle density $\rho(r)$ is given by its diagonal element as $[21,22]$

$\rho_{c}(r)=\left.\rho_{c}\left(r, r^{\prime}\right)\right|_{r=r^{\prime}}=\int_{0}^{\infty}|f(x)|^{2} \rho_{x}(r) d x$

In Eq. (15), $\rho_{x}(r)$ and $|f(x)|^{2}$ have the following forms $[2,3]$

$$
\begin{aligned}
& \rho_{x}(r)=\rho_{0}(x) \theta(x-|\vec{r}|) \\
& |f(x)|^{2}=\left.\frac{-1}{\rho_{0}(x)} \frac{d \rho_{c}(r)}{d r}\right|_{r=x}
\end{aligned}
$$

The weight function $|f(x)|^{2}$ of Eq. (17), determined in terms of the ground state $\rho_{c}(r)$, satisfies the following normalization condition $[2$, 3]

$$
n^{C D F M}\left(k:\left[\rho_{c}\right]\right)=\left(\frac{4 \pi}{3}\right)^{2} \frac{4}{Z} \times\left[6 \int_{0}^{V / k} \rho_{c}(x) x^{5} d x-\left(\frac{V}{k}\right)^{6} \rho_{c}\left(\frac{V}{k}\right)\right]
$$

with normalization condition

$$
Z=\int n^{C D F M}(k) \frac{d^{3} k}{(2 \pi)^{3}}
$$

while the experimental weight functions for ${ }^{70} \mathrm{Ge}$ and ${ }^{72} \mathrm{Ge}$ determined

$$
\begin{aligned}
& |f(x)|_{2 p F}^{2}=\frac{4 \pi x^{3} \rho_{0}}{3 A_{z}}\left(1+e^{\frac{x-c}{z}}\right)^{-2} \exp \left(\frac{x-c}{z}\right) \\
& |\mathrm{f}(\mathrm{x})|_{\mathrm{FB}}^{2}=-\sum_{\mathrm{v}} \frac{4 \pi \mathrm{x}^{3}}{3 \mathrm{Z}} \mathrm{a}_{\mathrm{v}}\left[\frac{\operatorname{Cos}(\mathrm{v} \pi \mathrm{x} / \mathrm{R})}{\mathrm{x}}-\frac{\operatorname{Sin}(\mathrm{v} \pi \mathrm{x} / \mathrm{R})}{\mathrm{x}(\mathrm{v} \pi \mathrm{x} / \mathrm{R})}\right]
\end{aligned}
$$

where the values of parameters $\mathrm{c}, \mathrm{z}$ and $a_{v}$ in above equations are taken from experimental data [24] while the constant $\rho_{0}$ is determined from the normalization of Eq. (5).

The elastic monopole charge form factors $F_{C O}(q)$ of the target nucleus are also expressed in the CDFM as [2, 3]: the two- parameter Fermi (2PF) and for ${ }^{74} \mathrm{Ge}$ and ${ }^{76} \mathrm{Ge}$ the Fourier- Bessel (FB), thus, yields respectively.

where

(20) is the Fermi-momentum distribution of
the system with density $\rho_{0}(x)$. By the system with density $\rho_{0}(x)$. By means of Eqs. (17), (19) and (20), an explicit form for the PMD is expressed in terms of $\rho_{c}(r)[2,3]$ as

$n_{x}(k)=\frac{4}{3} \pi x^{3} \theta\left(k_{F}(x)-|\vec{k}|\right)$

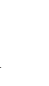


center of mass correction $f_{c m}(q)$ in the calculation requires multiplying the form factor of Eq. (25) by these corrections

Here, $f_{f s}(q)$ is considered as free nucleon form factor which is assumed to be the same for protons and neutrons [25]

$$
f_{f s}(q)=e^{-0.43 q^{2} / 4}
$$

The correction $f_{c m}(q)$ remove the spurious state arising from the motion of the center of mass when shell model wave function is used and is given by [25]

$$
f_{c m}(q)=e^{q^{2} b^{2} / 4 A}
$$

Multiplying the right hand side of Eq. (26) by these corrections yields:

$$
F_{C o}(q)=\frac{1}{Z} \int_{0}^{\infty}|f(x)|^{2} F(q, x) d x f_{f s}(q) f_{c m}(q) \text {. }
$$

It is important to point out that all physical quantities studied above in the framework of the CDFM such as $n(k)$ and $F_{C o}(q)$, are expressed in terms of the weight function $|f(x)|^{2}$. In the previous work $[2,3]$, the weight function was obtained from the NDD, extracted by analyzing elastic electronnuclei scattering experiments. In the present work, the theoretical weight function $|f(x)|^{2}$ is expressed, by introducing the derived CDD of Eq. (3) in to Eq. (17), as

$$
|f(x)|^{2}=\frac{8 \pi x^{4}}{3 Z b^{2}} \rho_{c}(x)-\frac{16 x^{4} e^{-x^{2} / b^{2}}}{3 Z b^{5} \pi^{1 / 2}}\left[\begin{array}{l}
\frac{11}{6} \alpha+\frac{5}{6}(Z-20-\beta) \\
+\left\{4-2 \alpha-\frac{4}{3}(Z-20-\beta)\right\}\left(\frac{x}{b}\right)^{2} \\
\left.+\frac{4}{35} \beta+\frac{2}{5}(Z-20-\beta)+\frac{2}{5} \alpha\right\}\left(\frac{x}{b}\right)^{4}
\end{array}\right]
$$

\section{Result and discussion}

In this study, the proton momentum distribution $n(k)$ and elastic electron scattering form factors, $F(q)$ are calculated by using CDFM, for some even $2 p$-1f shell nuclei, (such as: ${ }^{70} \mathrm{Ge}$, ${ }^{72} \mathrm{Ge},{ }^{74} \mathrm{Ge}$ and ${ }^{76} \mathrm{Ge}$ ) isotopes. The distribution $n^{C D F M}(k)$ of Eq. (21) was calculated by means of the CDD which was obtained firstly from theoretical consideration, like in Eq. (3). And then secondly from experimental data (such as $2 \mathrm{PF}$ for ${ }^{70} \mathrm{Ge}$ and ${ }^{72} \mathrm{Ge}$ [24] and FB for ${ }^{74} \mathrm{Ge}$ and $\left.{ }^{76} \mathrm{Ge}[10]\right)$. The size parameters $b$ is choice in such a way so as to imitate the experimental root mean square (rms) charge radii of nuclei. The values of $\alpha$ are determined by Eq. (8). The values of $b$ and $(\alpha, \beta)$ simultaneously with value of the central densities $p_{e x}(0)$ and the root mean square charge radii $\left\langle r^{2}\right\rangle_{\exp }^{1 / 2}$ for ${ }^{70} \mathrm{Ge},{ }^{72} \mathrm{Ge},{ }^{74} \mathrm{Ge}$ and ${ }^{76} \mathrm{Ge}$ nuclei are present in Table 1 and 2. 
Table 1: The value of various parameters employed to CDD.

\begin{tabular}{|c|c|c|c|}
\hline Nucleus & $\begin{array}{c}\text { Type of } C D D \\
{[10]}\end{array}$ & $\begin{array}{l}\rho_{\exp }(0) \\
\left(f m^{-3}\right)[10] \\
P W \text { eq.(7) }\end{array}$ & $\begin{array}{c}\left\langle r^{2}\right\rangle_{\text {exp }}^{1 / 2} \\
(\mathrm{fm})[10]\end{array}$ \\
\hline${ }^{70} \mathbf{G e}$ & $F B$ & 0.07609876 & 4.043 \\
\hline${ }^{72} \mathbf{G e}$ & $F B$ & 0.07443733 & 4.060 \\
\hline${ }^{74} \mathbf{G e}$ & $F B$ & 0.07260928 & 4.075 \\
\hline${ }^{76} \mathbf{G e}$ & $F B$ & 0.07106188 & 4.081 \\
\hline
\end{tabular}

Table 2: Calculated parameters used in Eq. (3), To calculate CDD and occupation number in $1 f-2 p$ of nucleus and the calculated $\left\langle r^{2}>_{\text {cal }}^{1 / 2}\right.$.

\begin{tabular}{|c|c|c|c|c|c|c|c|}
\hline Nucleus & $\mathrm{b}$ & $\alpha$ & $\beta$ & $\begin{array}{c}\text { Occupation } \\
\text { No. of } 2 \mathrm{~s} \\
(2-\alpha)\end{array}$ & $\begin{array}{c}\text { Occupation } \\
\text { No. of 1f } \\
\beta\end{array}$ & $\begin{array}{c}\text { Occupation } \\
\text { No. of 2p } \\
(Z-20-\beta+\alpha\end{array}$ & $\left\langle r^{2}\right\rangle_{\text {cal }}^{1 / 2}$ \\
$(6)$
\end{tabular}

In Fig. 1, explore the dependence of the CDD (in $\mathrm{fm}^{-3}$ ) on $\mathrm{r}$ (in $\mathrm{fm}$ ) for ${ }^{70} \mathrm{Ge}$ [Fig. 1(a)], ${ }^{72} \mathrm{Ge}$ [Fig. 1(b)], ${ }^{74} \mathrm{Ge}$ [Fig. 1(c)] and ${ }^{76} \mathrm{Ge}[$ Fig. 1(d)] nuclei. The solid and dotted curves are the measured charge density distributions of the treated nuclei by using Eq. (3) when $\alpha \neq 0$ and $\alpha=0$, respectively while the solid circles correspond to the experimental data [24]. It is noticeable that the dotted curves distribution are poor agreement with the experimental data, particular for small $\mathrm{r}$. Introducing the parameters $\alpha$ and $\beta$ (i.e., taking into account the higher orbitals) into our calculations leads to a good agreement with the experimental data as demonstrated by the solid curves. 

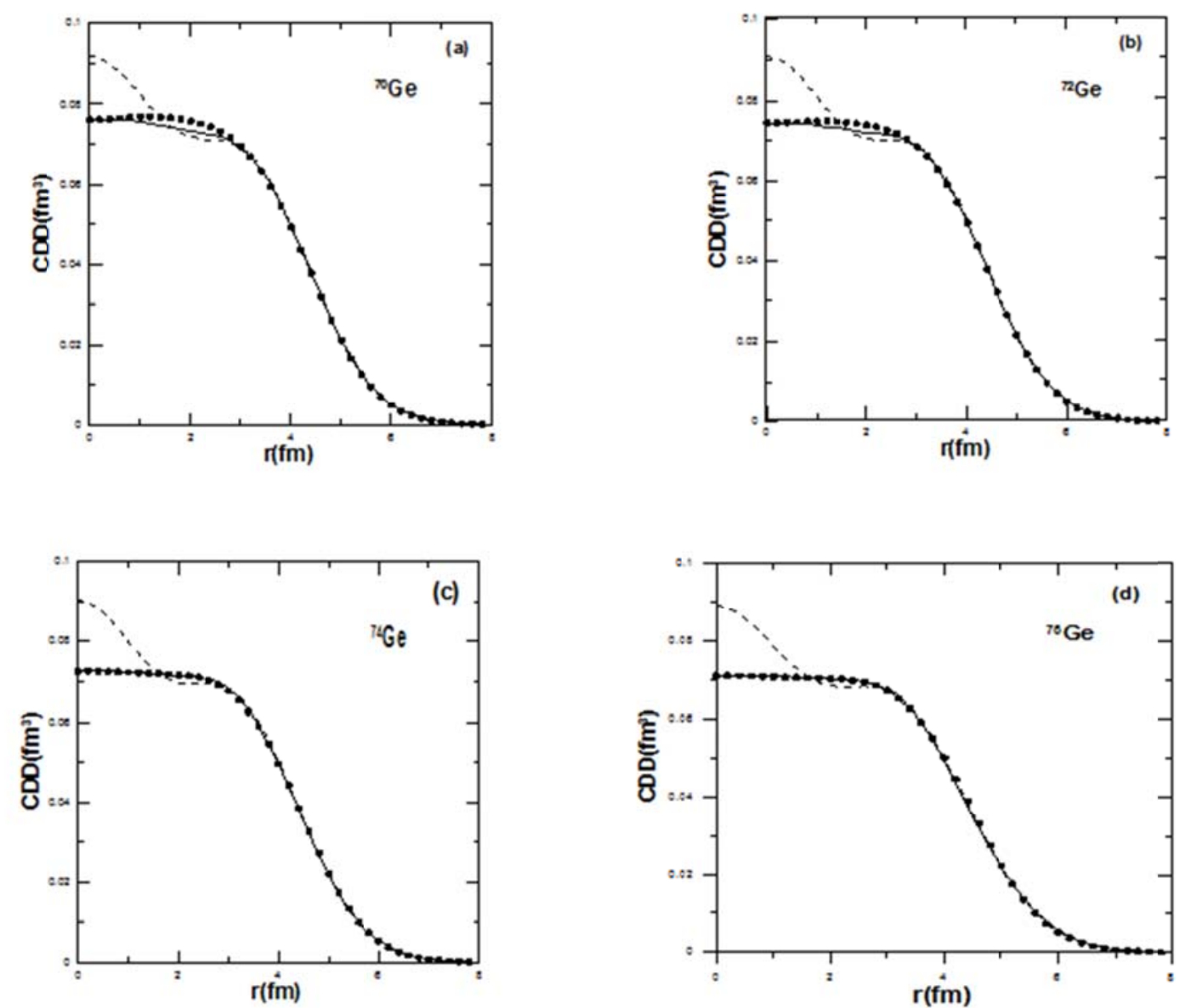

Fig. 1: The dependence of the CDD on $r$ for $(a)^{70} G e,(b)^{72} G e,(c)^{74} G e$ and $(d)^{76} G e$ nuclei. the solid and dotted curves are the calculated CDD of the treated nuclei by using Eq. (3) when $\alpha \neq 0$ and $\alpha=0$, respectively whereas the solid circles are those fitted to the experimental data of Fourier-Bessel (FB) CDD [24].

In Fig. 2, we display the dependence of the $n(k)$ (in $\mathrm{fm}^{3}$ ) on $\mathrm{k}$ (in $\mathrm{fm}^{-1}$ ) for ${ }^{70} \mathrm{Ge}$ [Fig. 2(a)], ${ }^{72} \mathrm{Ge}$ [Fig. 2(b)], ${ }^{74} \mathrm{Ge}$ [Fig. 2(c)], and ${ }^{76} \mathrm{Ge}$ [Fig. 2(d)] nuclei. The long-dashed curves correspond to the PMD's of Eq. (10) evaluated by the shell model calculation used the single particle harmonic-oscillator wave function in the momentum space. The solid circles symbols and solid curves correspond to the PMD's obtained by CDFM of Eq. (21) employing the experimental and theoretical CDD, respectively. It is evident that the behavior of the dash distribution curves estimated by the shell model is in contrast with distributions imitated by the CDFM. The significant property of the long-dashed distribution is the steep slope mode, when $\mathrm{k}$ is increases. This behavior is in disagreement with our studies [2, 3, 26, 27] and it is attributed to the fact that the ground state shell model wave function given in terms of Slater determinant does not take into account the major effect of the short range dynamical correlation functions. Hence, the short range repulsive features of the nucleonnucleon force are responsible for the high momentum behavior of the PMD $[26,7]$. The property of long-tail behavior obtained by the CDFM, which is $\mathrm{i} \mathrm{n}$ agreement with the studies $[2,3,26,27]$, is connected to the presence of high densities $\rho_{x}(r)$ in the decomposition of Eq. (15), though 
their fluctuation functions $|f(x)|^{2}$ are small. The PMD of $\left({ }^{70} \mathrm{Ge},{ }^{72} \mathrm{Ge}\right)$ nucleus present in Figs. 2 (a) and 2 (b), respectively, shows quite well agreement between the calculated data (the solid curve) and the experimental data (solid circles) up to $\mathrm{k}=2.2 \mathrm{fm}^{-1}$, while beyond this region they shows an explicit deviation between them. Besides, This deviation in PMD at large $\mathrm{k}$ may be interpreted by the deviation between the calculated charge density distribution of the $\left({ }^{70} \mathrm{Ge}\right.$ and ${ }^{72} \mathrm{Ge}$ ) nuclei and those of the experimental two parameters Fermi by used the charge density equation: $\rho(r)=\rho_{0} /(1+\exp ((r-c) / z))$ where their parameter is listed in Table 3 [24]. Since this deviation affect greatly
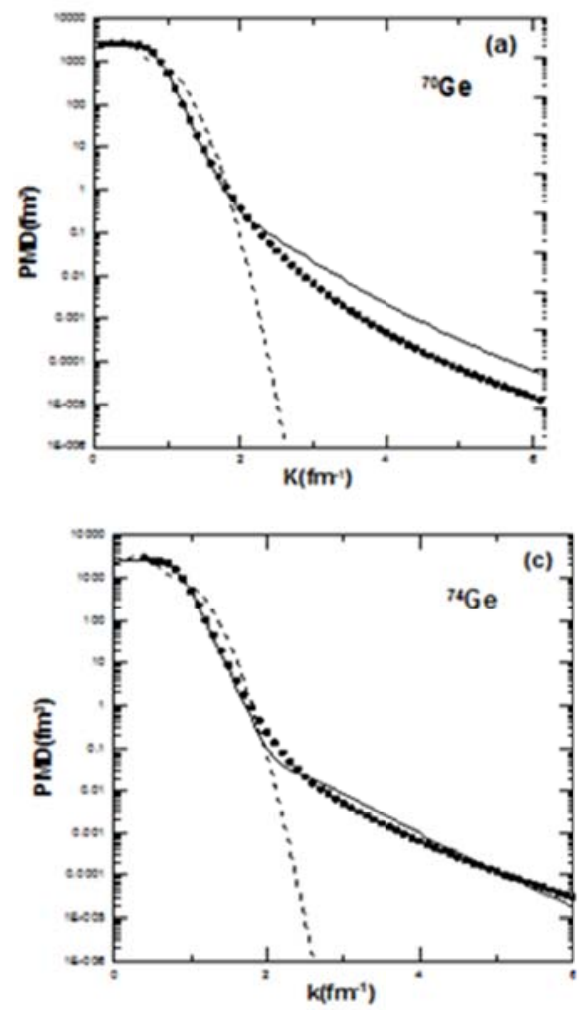

Fig. 2: The dependence of PMD on $k$ for $(a)^{70} \mathrm{Ge},(b)^{72} \mathrm{Ge}$, (c) ${ }^{74} \mathrm{Ge}$ and $(\mathrm{d}){ }^{76} \mathrm{Ge}$ nuclei. The long-dashed curves are the calculated PMD of Eq. (10) obtained by the shell model calculation using the single particle harmonic oscillator wave functions in the momentum representation. The solid circles symbols and solid curves distributions are the calculated PMD obtained in terms of the CDFM of Eq.(21) using the experimental 2 PF of Eq.(23) and theoretical weight function for ${ }^{70} \mathrm{Ge}$ and ${ }^{72} \mathrm{Ge}$ of Eq. (30), respectively, and for ${ }^{74} \mathrm{Ge}$ and ${ }^{76} \mathrm{Ge}$ by using the experimental FB of Eq.(24) and theoretical weight function respectively. 
Table 3: The value of various parameters employed to CDD of $2 \mathrm{PF}$ for ${ }^{70} \mathrm{Ge}$ and ${ }^{72} \mathrm{Ge}$.

\begin{tabular}{|c|c|c|c|c|}
\hline Nuclei & $\begin{array}{c}\text { Type of } \\
C D D[24]\end{array}$ & $\begin{array}{l}c(f m) \\
(24)\end{array}$ & $\begin{array}{l}z(f m) \\
(24)\end{array}$ & $\begin{array}{l}\left\langle\mathrm{r}^{2}\right\rangle_{\text {exp }}^{1 / 2}(\mathrm{fm}) \\
(24)\end{array}$ \\
\hline${ }^{70} \mathbf{G e}$ & $2 \mathrm{pF}$ & $4.44(2)$ & $0.585(7)$ & $4.07(2)$ \\
\hline${ }^{72} \mathbf{G e}$ & $2 \mathrm{pF}$ & $4.45(2)$ & $0.573(7)$ & $4.05(3)$ \\
\hline
\end{tabular}

The elastic electron scattering charge form factors for the considered nuclei are calculated in the framework of the (CDFM) through introducing the theoretical weight functions $|f(x)|^{2}$ of Eq. (30) into Eq. (29). In Fig. 3, we present the dependence of the form factors $F(q)$ on the momentum transfer $\mathrm{q}$ (in $\mathrm{fm}^{-1}$ ) for ${ }^{70} \mathrm{Ge}$ [Fig. 3(a)], ${ }^{72} \mathrm{Ge}$ [Fig. 3(b)], ${ }^{74} \mathrm{Ge}$ [Fig. 3(c)] and ${ }^{76} \mathrm{Ge}$ [Fig. 3(d)] nuclei, where the solid circles are representing experimental data [10]. In Fig. 3(a) ${ }^{70} \mathrm{Ge}$ and Fig. 3(b) ${ }^{72} \mathrm{Ge}$ shows that the
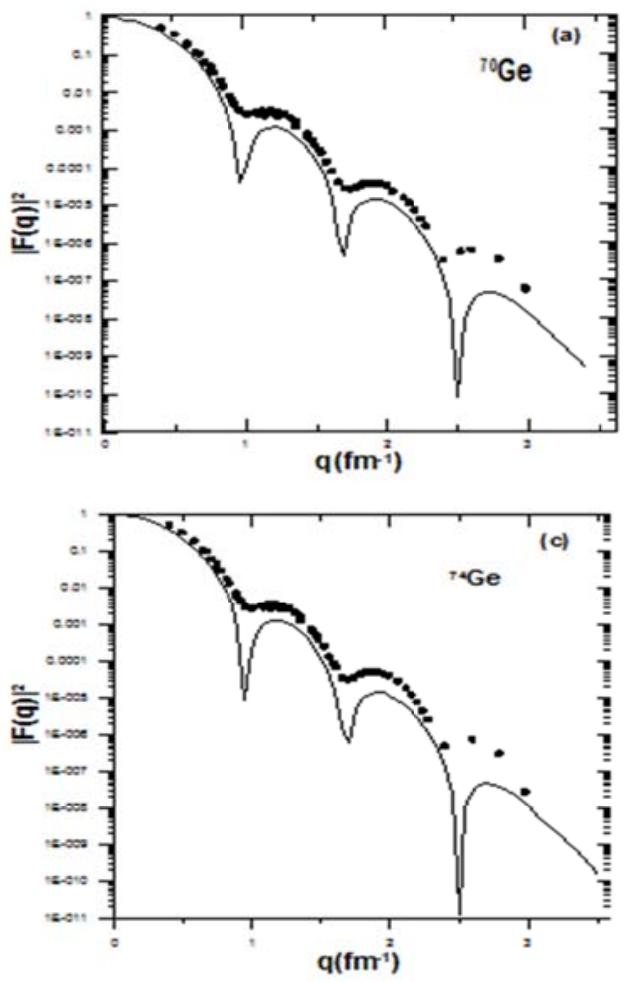

diffraction minima and maxima of the considered nuclei are reproduced in the correct places. While in Fig. (3c) ${ }^{74} \mathrm{Ge}$ and Fig. (3d) ${ }^{76} \mathrm{Ge}$ the third diffraction minimum of experimental data [10] is shifted slightly by about $\mathrm{q}=0.5 \mathrm{fm}^{-1}$ from the calculated one demonstrated by the solid curve which is located at $\mathrm{q}$ $=2.5 \mathrm{fm}^{-1}$. In all these Fig. 3, both the behavior and the magnitudes of the calculated form factors of these nuclei are in reasonable agreement with those of the experimental data.
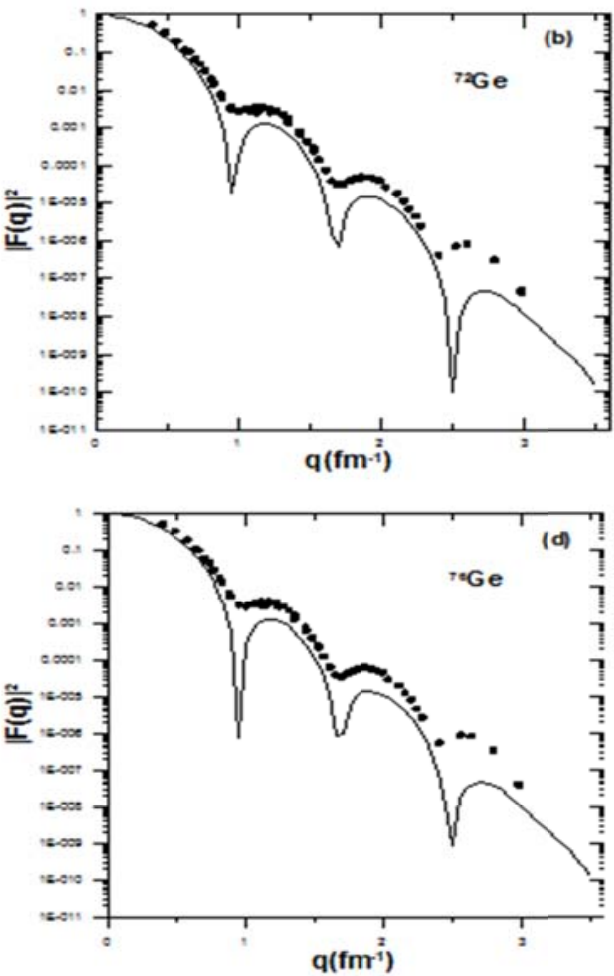

Fig. 3: Dependence of the charge form factors on $q$ for (a) ${ }^{70} \mathrm{Ge}$, (b) ${ }^{72} \mathrm{Ge}$, (c) ${ }^{74} \mathrm{Ge}$ and (d) ${ }^{76} \mathrm{Ge}$ nuclei. The solid curve is the calculated $F(q)$ of the Eq. (25). The filled circle symbols are the experimental data, taken from Ref. [10]. 


\section{Conclusions}

The (PMD) and elastic electron scattering form factors, calculated in the framework of the (CDFM), are expressed by means of the weight function $|f(x)|^{2}$. The weight function, which is connected with the local density $\rho(r)$, was determined from experiment and from theory. The feature of the long-tail behavior of the (PMD) is obtained by both theoretical and experimental weight functions, which is in agreement with the other studies [2, 3, 26, 27] and is related to the existence of high densities $\rho_{x}(r)$ in the decomposition of Eq. (15), though their weight functions are small. The experimental form factors for elastic electron scattering from ${ }^{70} \mathrm{Ge},{ }^{72} \mathrm{Ge}$, ${ }^{74} \mathrm{Ge}$ and ${ }^{76} \mathrm{Ge}$ nuclei are well reproduced by the monopole form factors. It is noted that the theoretical (CDD) of Eq. (3) employed in the determination of the theoretical weight function of Eq. (30) is capable of reproducing information about the (PMD) and elastic form factors.

\section{Acknowledgment}

We wish to thank Professor G. K. Mallot for providing us with the unpublished experimental data for the Germanium isotopes.

\section{Reference}

[1] P. M. Morse and H. Feshbach, Methods of Theoretical Physics. Part 2, McGraw-Hill book Company, 1953, New York.

[2] A. N. Antonov, P. E. Hodgson, I. Zh Petkov., Nucleon Momentum and Density Distribution in Nuclei. Clarendon Press, Oxford, (1988) 1165.

[3] A. N. Antonov, V. A. Nikolaev, I. Zh Petkov., Physik, A297 (1980) 257260.

[4] R. D. Amado, Phys. Rev., C14 (1976) 1264.
[5] V. I. Komarov, G. E. Kosarey, H. Muler, D. Netzband, T. Stiehler, Phys. Lett., B 69 (1977) 37.

[6] R. A. Ridha, Elastic electron scattering form factors and nuclear momentum distributions in closed and open shell nuclei, M.Sc. Thesis, (2006) University of Baghdad.

[7] M. Traini and G. Orlandini, Physik Z., A321 (1985) 479-484.

[8] M. Dal Ri, S. Stringari, O. Bohigas, Nucl. Phys., A376 (1982) 81.

[9] T. Y. Donnelly and J. D. Walecka Ann., Rev. Nucl., Part. Sci. 25 (1975) 329.

[10] G. K. Mallot, Electron Scattering of Germanium and Muonic Atoms of Krypton as a Contribution to the Systematics of the Nuclear Charge Densities, Ph.D., thesis (1984) University of Mainz.

[11] G. Fricke, C. Bernhardt, K. Heiling, L. A. Schaller, L. Schellenberg, E.B. Shera, C. W. Jager, Nucl. Data Table, (1995) 60 -177.

[12] J Heisenberg and H P Blok Ann., Rev. Nucl., Part. Sci. 33 (1983) 569.

[13] R. Hofstadter, lectron Scattering and Nuclear Structure Rev. Mod. Phys. 28, (1956) 214.

[14] A. H. Wapstra, G. Audi, R. Hoekstra, Data Nucl., Data Tables 39, (1988) 281.

[15] Z Wang and Z. Ren, Phy. Rev., C 71 (2005) 5432.

[16] Z. Wang, Z. Ren, Y. fan, Phy. Rev., C 73 (2006) 14610-1.

[17] X Roca- Maza, M Centelles, F. salvat, X.Vinas, Phys. Rev., C 78 (2008) 44332.

[18] S. Karataglidis and K. Amos, Phys. Lett., B 650 (2007) 148.

[19] Y. Chu, Z. Ren, T. Dong, Z. Wang, Phys. Rev., C 79 (2009) 443131

[20] A. A. Al-Rahmani and $\mathrm{H}$. Hussein, Iraqi Journal of Science, 55 4B, (2014) 1868. 
[21] A. K. Hamoudi, M. A. Hassan, A. R. Ridha, PRAMANA- Journal of Physics Indian Academy of Sciences, 78, 5 (2012) 737-748.

[22] A. A. AL-Rahmani, International Journal of Science and Research, IJSR, 5, 1, (2016) 741. / A. A. AL-Rahmani, Indian J. Phys. (2016).

[23] N. G. Shevchenko, A. A. Khomich, Yu. Buki A., V. N. Polishchuk, B. V. Mazanko, Yu. A. Kasatkin, Sov. Nucl Phys., 27 (1978) 159.
[24] H. De Vries, C. W. De Jager, C. De. Vries. At. Data Nucl. Data Tables. 36, (1987) 495.

[25] B. A. Brown, R. A. Radhi, B. H. Wildenthal. Phys. Rep., 101, 5 (1983) 313-358

[26] H.C Moustakidis and S.E. Massen, Phys. Rev., C 62 (2000) 34318-1 34318-7.

[27] M. Dal Ri, S. Stringari, O. Bohigas. Nucl. Phys., A376 (1982) 81. 